\title{
Mission Design of an Aperture-Synthetic Interferometer System for Space-Based Exoplanet Exploration
}

\author{
Feida Jia $\mathbb{D}^{1,2}$ Xiangyu Li, ${ }^{1,2}$ Zhuoxi Huo, $^{3}$ and Dong Qiao ${ }^{1,2}$ \\ ${ }^{1}$ School of Aerospace Engineering, Beijing Institute of Technology, Beijing 100081, China \\ ${ }^{2}$ Key Laboratory of Autonomous Navigation and Control for Deep Space Exploration, Ministry of Industry and \\ Information Technology, Beijing 100081, China \\ ${ }^{3}$ Qian Xuesen Laboratory of Space Technology, Beijing 100094, China
}

Correspondence should be addressed to Xiangyu Li; lixiangy@bit.edu.cn

Received 30 August 2021; Accepted 21 January 2022; Published 17 February 2022

Copyright (C) 2022 Feida Jia et al. Exclusive Licensee Beijing Institute of Technology Press. Distributed under a Creative Commons Attribution License (CC BY 4.0).

\begin{abstract}
In recent years, exoplanet detection has become the technological frontier in the field of astronomy, because it provides evidence of the origin of life and the future human habitable exoplanet. Deploying several satellites to form an aperture-synthetic interferometer system in space may help discover "another Earth" via interferometry and midinfrared broadband spectroscopy. This paper analyzes a space-based exoplanet exploration mission in terms of the scientific background, mission profile, trajectory design, and orbital maintenance. First, the system architecture and working principle of the interferometer system are briefly introduced. Secondly, the mission orbit and corresponding transfer trajectories are discussed. The halo orbit near the Sun-Earth L2 (SEL2) orbit is chosen as the candidate mission orbit. The low-energy transfer via stable invariant manifold with multiple perigees is designed, and the proper launch windows are presented. A speed increment less than $10 \mathrm{~m} / \mathrm{s}$ is imposed for each transfer to achieve the insertion of the halo orbit. Finally, the tangent targeting method (TTM) is applied for high-precision formation maintenance with the whole velocity increments of less than $5 \times 10^{-4} \mathrm{~m} / \mathrm{s}$ for each spacecraft when the error bound is $0.1 \mathrm{~m}$. The overall fuel budget during the mission period is evaluated and compared. The design in this paper will provide technical support and reliable reference for future exoplanet exploration missions.
\end{abstract}

\section{Introduction}

The search for extraterrestrial life and exploration of "another Earth" is an eternal theme for humans and inspires generations of planetary scientists. It not only improves our understanding of the formation and evolution of planets during the formation of a star system but also helps scientists to investigate the possible conditions and criteria of the existence of life. The 2019 Nobel Prize in Physics was awarded for the first discovery of an exoplanet near a Sunlike star, in recognition of the work "for contributions to our understanding of the evolution of the universe and Earth's place in the cosmos," one half of which was awarded to James Peebles "for theoretical discoveries in physical cosmology" and the other jointly to Michel Mayor and Didier Queloz "for the discovery of an exoplanet orbiting a solar- type star" [1]. In the past 30 years, scientists have discovered more than 4,000 exoplanets, but exploration still has a long way to go. Due to the far distance, searching for exoplanets requires high sensitivity and high resolution. The spacebased telescope can eliminate the interference of the Earth's atmosphere on observations and becomes a trend in exoplanet exploration. Several telescopes have been launched into orbit such as the Kepler space telescope and TESS (Transiting Exoplanet Survey Satellite). Limited by the launch capability, the size of a single space-based telescope cannot be as large as required for further explorations than in current missions which employ methods such as transit and radial velocities. As a next-generation telescope configuration, the array telescope is expected to realize the cross-generation of the telescope system so that an Earth-like exoplanet near a Sun-like host star could be detected and characterized via 
direct imaging, provided that technological challenges can be overcome, and opens a new era of high-resolution astronomical observation [2].

The array telescope will form a long-baseline interferometer in space, which largely improves the observation resolution. Several mission concepts of the array telescope for exoplanet detection have been proposed in the past twenty years. For example, the TPF mission [3] and Darwin mission [4] are proposed by NASA and ESA, respectively. They both use multiple (3-5) satellites to form a formation of fixed architecture near the halo orbit to search for exoplanet life. NASA's Stellar Imager (SI) project [5] plans to deploy 2030 satellites to form formations near the beam axis in the Lissajous orbit around the Sun-Earth libration point, and each satellite is equipped with a mirror with a diameter of $1 \mathrm{~m}$ to perform 0.1-milliarcsecond (mas) spectral imaging of the surface of stars and the entire universe. Encouraged by the significance of exoplanet exploration, in 2019, CASC also proposed a habitable exoplanet exploration mission by array telescope called the MEAYIN mission [6].

The essence of the mission in this study is formation flying around the libration point. Many researchers are devoted to this theme in recent years. The concept of formation flying around equilibrium was first proposed by Barden and Howell [7], and they gave the concept of using the central manifold of the halo orbits to realize a natural bounded formation. Héritier and Howell $[8,9]$ defined the regions above as low drift regions, expressed them in the form of secondorder surfaces, and studied the small-size formations near the halo orbit and the large-size formations on the Lissajous orbit. It is found that the initial spherical surface will become an ellipsoidal one, while it will become smaller as the distance from the Earth decreases. Several studies [10-12] are aimed at the design of formation flying to obtain the stable configuration in CRTBP or bicircular model (BCM). Meanwhile, the solar sail could be applied to achieve a synchronized formation tracking with a virtual leader. With this background, this paper elaborates the mission analysis in terms of the scientific background, mission profile, trajectory design, and orbital maintenance.

The structure of the rest of this paper is as follows. In Section 2, the architecture and principles of exoplanet exploration are discussed. The mission constraints and accuracy requirements on formation flight are given. In Section 3, the candidate mission orbit is selected, and the corresponding transfer trajectory is designed under the ephemeris model. Section 4 deals with the formation configuration maintenance. The high-precision impulsive-based control method of the tangent targeting method (TTM) is applied to satisfy the stability region constraints. Finally, Section 5 draws the conclusion.

\section{Mission Architecture and Principle of Interferometry}

2.1. Observation Demand and Architecture of Array Telescope. The array telescope mission intends to observe various types of celestial bodies such as extrasolar habitable planets, solar system celestial bodies, protoplanetary disks, and active galactic nuclei. Searching for and characterizing habitable exoplanets in our solar system's neighbors (within 65 light-years) place a high demand on observations including the following:

(1) High spatial resolution. The star-planet angular distance is better than 0.01 arcsec 65 light-years away from the Sun.

(2) High contrast. The brightness of planets and stars differs by at least 7 orders of magnitude in the midinfrared band.

(3) High sensitivity. The brightness of the planet in the signal dominant band is less than 3 photons $/ \mathrm{sec} / \mathrm{m}^{2}$.

(4) Wide spectral range. Indirect observation in the near-infrared band of 1 to $5 \mu \mathrm{m}$ and direct observation in the near-infrared band of 1 to $13 \mu \mathrm{m}$.

The spatial resolution of the interferometer is related to the accuracy of path difference control, baseline length, and integration time. Therefore, the baseline of the interferometer should be designed according to the resolution requirement. Meanwhile, the control accuracy of the optical path difference and integration time should match the baseline range.

One of the difficulties in direct imaging of extragalactic habitable planets is the high contrast between the brightness of Earth-like planets and their star. It is difficult to suppress the stellar radiation effectively in the midinfrared band by using a single aperture optical telescope, while zero-order interferometry in multisatellite formation can theoretically meet the requirements of high contrast and high resolution at the same time. In this project, the array telescope system consists of a collector in the center and four detectors evenly distributed around. The architecture of the on-orbit observation scene is shown in Figure 1. The dash lines between the detectors and the collector represent the optical communication links.

The array telescope system includes a detector system and a collector system, both of which are divided into two parts which are the platform and the load. The platform is divided into five function modules: the structure and organization, thermal control, position control, and energy and information management. The load of the detector system includes collecting optical system and telescope detectors, while the load of the collector system consists of combining the optical system and interferometric measuring device.

2.2. Principle of Interferometry. Interference arrays are usually aimed at the star to be observed when they are in operation. At this point, the star light propagates along the principle optical axis of the array and reaches the telescope, while the planetary light arrives at a small angle $\theta$ away from the principle optical axis of the array. For ordinary binary interference systems, the light incident along the principle optical axis propagates out of the beam through the left and right arms of the interference array with the same 


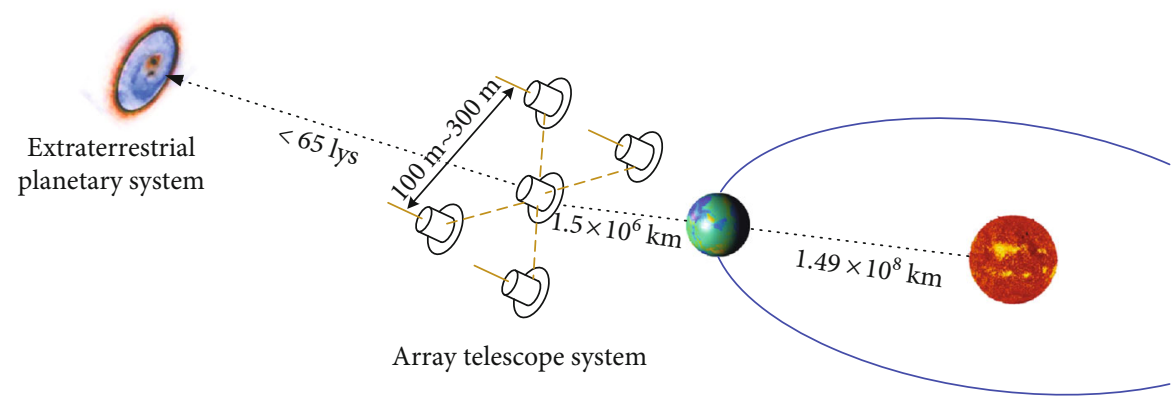

FIgURE 1: On-orbit observation scene of an array telescope system.

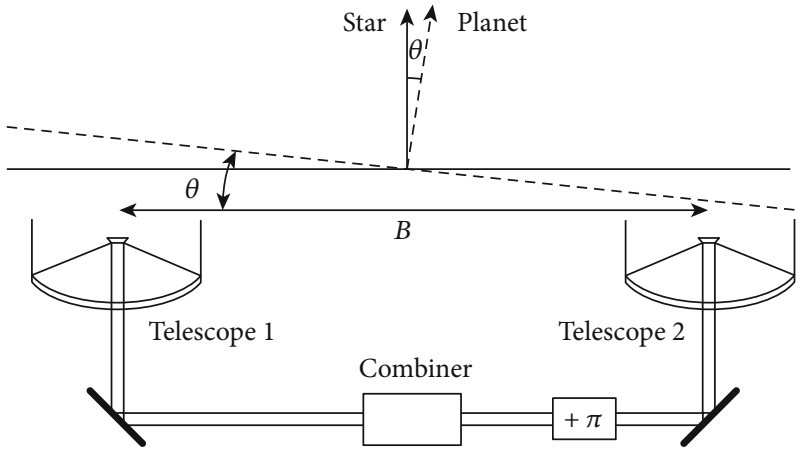

Figure 2: Schematic diagram of two-element nulling interferometer [13].

optical path (or optical path with difference of integer times of the wavelength), so the interference fringes are mutually reinforcing at the zero-order position.

The two-element nulling interferometer introduces the phase delay of $\pi$ (half wavelength) in one arm (as shown in Figure 2), so the interference fringes formed by the light incident along the principle optical axis offset each other at zero-order. There can be mutually reinforcing bright fringes at zero-order of the interference fringes, which are formed by two kinds of light along and slightly deviated from the principle optical axis.

The four-element nulling interferometer is proposed on the basis of the usual two-element nulling interferometer. By introducing the inverse signal with another pair of interference beams, the nulling range is greatly widened, and the nulling depth is improved by more than three orders of magnitude, which provides great help for the detection of exoplanets, especially Earth-like stars. The optical path of the four-element nulling interferometer is shown in Figure 3. $O_{i}(i=1,2,3,4)$ are the interference lights in four positions.

It combines two two-element nulling interferometers with different spacing (the spacing of the internal and external pair are $2 \mathrm{~s}$ and $4 \mathrm{~s}$, respectively) and adjusts the beam intensity ratio so that the amplitude of the external pair is exactly half of that of the internal one. The interference amplitudes of these two pairs cross the zero point with the same value but opposite sign, so as to achieve high-order limitation.

Based on the characteristics of the observation demand and the principle of interferometry, the general requirements for the array telescope system are shown in Table 1 .

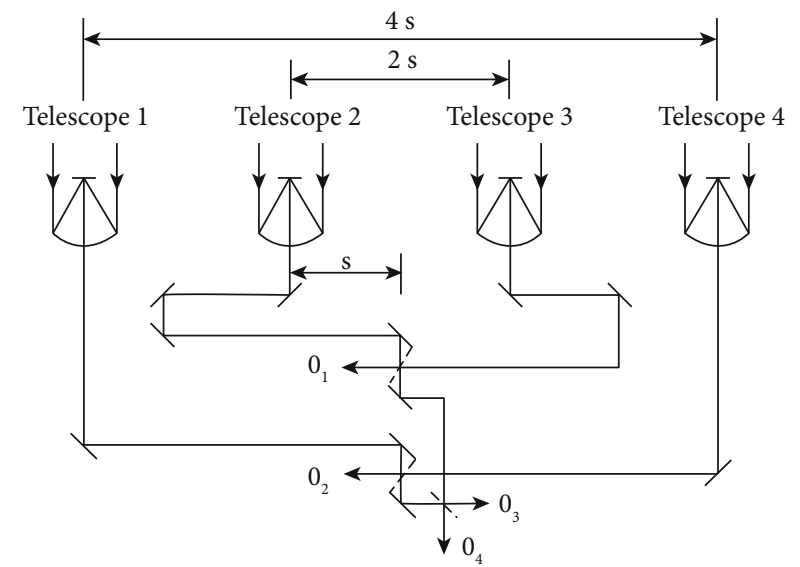

FIgURE 3: Schematic diagram of four-element nulling interferometer.

TABLE 1: General path-dependent technical requirements.

\begin{tabular}{lc}
\hline Index & Value \\
\hline Inertia pointing accuracy & $\leq 2^{\prime \prime}(3 \sigma)$ \\
Formation baseline range & $40 \mathrm{~m}-300 \mathrm{~m}$ \\
Relative position control accuracy & $\leq 10 \mathrm{~cm}(3 \sigma)$ \\
Load platform position accuracy & $\leq 1 \mathrm{~mm}(3 \sigma)$ \\
Main optical axis pointing accuracy & $\leq 0.1^{\prime \prime}(3 \sigma)$ \\
Transfer and maintenance velocity increment & $\leq 1.6 \mathrm{~km} / \mathrm{s}$ \\
\hline
\end{tabular}

These requirements will be considered in the following trajectory design and maintenance.

\section{Mission Orbit Selection and Transfer Trajectory Design}

3.1. Mission Orbit Selection for Exoplanet Exploration. The mission orbit is crucial for the exoplanet exploration by using an array telescope. The ideal mission orbit should keep away from the electromagnetic interference in the vicinity of the Earth and operate in a relatively clean dynamic environment to reduce the magnitude and frequency of orbit maintenance. There are two kinds of candidate mission orbits for the mission, the Sun-Earth libration orbit and the Earthtrailing orbit. 
TABLe 2: Comparison and analysis of the halo and Earth-trailing orbit.

\begin{tabular}{lcc}
\hline Index & Halo orbit & Earth-trailing orbit \\
\hline Shadow occlusion & No occlusion & No occlusion \\
Cost of orbit insertion & Relatively low & Relatively high \\
Distance to earth & 1.5 million km & 1 million to 10 million km \\
Earth communication coverage & Stable & Gradually increase \\
Velocity increment of coverage & Always visible & Always visible \\
Maintenance frequency & Relatively small & Relatively large \\
\hline
\end{tabular}

Due to the properties of three-body dynamics, the SunEarth libration orbit can maintain a relatively fixed position with the Earth and the Sun, which is convenient for tracking and control. The spacecraft can transfer into orbit via stable invariant manifolds, which requires nearly zero energy after launch. Meanwhile, the array telescope can stay in the libration orbit for a long time with very little fuel. By choosing the amplitude of the orbit, the array telescope system can avoid an eclipse permanently. Therefore, the array telescope system can have a long working life.

The Earth-trailing orbit is the other ideal mission orbit, which stays close to the Earth although it undergoes a slow drift away from the Earth's orbit. The Earth-trailing orbit can provide full-time lighting conditions and a stable thermal environment. However, the spacecraft on the Earthtrailing obit will continuously drift away from the Earth, which stresses the communications system. Moreover, it requires more launch energy to send the space-based array telescope to the mission orbit, and the spacecraft must perform correction maneuvers to stay at the desired position. Based on the above reasons, the Sun-Earth libration orbit is chosen as the mission orbit for the space-based array telescope system.

There are many kinds of periodic and quasiperiodic orbits near the SEL2 point including planar and vertical Lyapunov orbits, halo orbits, Lissajous orbits, and axial orbits. Planar Lyapunov orbits always move in the $X Y$ plane of the Sun-Earth rotating frame, while the vertical orbits are " 8 "-shaped and move mainly along the $Z$-axis.The Lissajous orbits are nonclosed orbits, of which the characteristic frequencies in the $X Y$ plane and $Z$ direction are independent. The projection of the Lissajous orbit in the $X Y$ plane is approximately elliptical, but the orbital plane twists in each period. The halo orbits are closed curves in threedimensional space. The in-plane frequency and out-plane frequency of the halo orbits are coupled. Meanwhile, several quasiperiodic orbits surround the periodic orbits and form a two-dimensional torus, which provides more flexible options for mission design.

Both the plane and the vertical Lyapunov orbits will suffer from the eclipse, which affects the performance of the telescope system. The Lissajous orbit also passes through the $X$-axis nearby region in a long run. Moreover, the stability of large amplitude Lissajous orbits is poor. In the meantime, the simulation shows that when the amplitude of the halo orbit is larger than $10,000 \mathrm{~km}$, the spacecraft can avoid

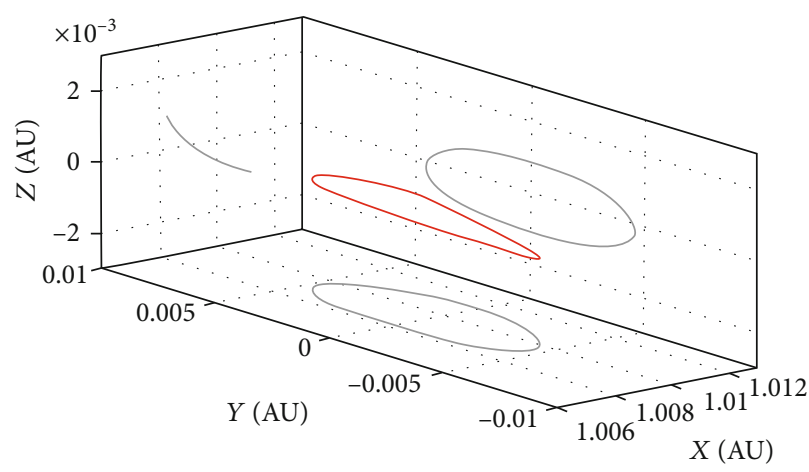

FIgURE 4: Mission orbit for exoplanet exploration in the rotating frame.

the occlusion of the Earth and achieve full-time light. Therefore, the halo orbits are more suitable as the mission orbit for the space-based array telescope system [14]. A comparative analysis of the halo orbit and Earth-trailing orbit is given in Table 2. Considering the stability and fuel consumption, the amplitude of the halo orbit is selected to be about $150,000 \mathrm{~km}$ in the $Z$ direction. The mission orbit in the rotating frame is shown in Figure 4.

\subsection{Transfer Trajectory Design for Exoplanet Exploration.} Based on the selected periodic orbit, the design of the corresponding transfer trajectory is investigated. The stable invariant manifold of the periodic orbit is used to find the low-energy transfer opportunity. The transfer trajectory is designed as follows. Firstly, based on the circular restricted three-body problem (CRTBP), the stable manifolds of the target mission orbit are generated at different phase angles, and the branch approaching the Earth is selected. Then, the Poincare map is selected according to the perigee state constraint. Afterward, the corresponding manifold that satisfies the height constraint of the parking orbit is chosen as the initial guess of the transfer trajectory. Figure 5 shows the chosen halo orbit and its stable manifold extending to the perigee truncated by the Poincare map. Since the stable manifold and the halo orbit are designed based on the CRTBP, the nominal transfer trajectory has a large error, when considering the orbital eccentricity of the Earth and the perturbation forces such as the gravitational force of the Moon. Finally, the conventional differential correction 


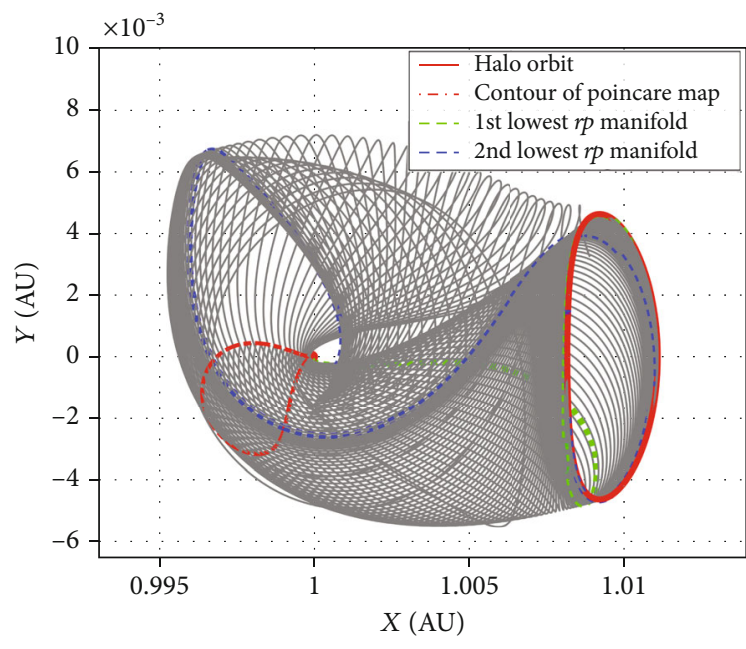

Figure 5: Stable manifolds extending to perigee of Earth.

algorithm is adopted to modify the transfer trajectory in the high-fidelity model.

Considering the weight and size of the satellite, it is difficult to transport five satellites to the mission orbit simultaneously, so three launches are planned to deploy four detectors and one collector to the mission orbit. The collector and one detector are launched for the first time, two detectors for the second time, and the remaining detector for the last time. Since the size of the formation is much smaller than the amplitude of the halo orbits, the specific configuration of the formation can be ignored in the transfer orbit design.

If we choose the altitude of parking orbit as $200 \mathrm{~km}$, the proper stable manifold in CRTBP corresponds to the phase angle of the halo orbit in intervals $\left[290^{\circ} 330^{\circ}\right]$ for the first perigee and the transfer time is between 170.2 and 173.5 days. For the second perigee, the phase angle $\left[170^{\circ} 220^{\circ}\right]$ and the transfer time are about 243 days. It should be noted that $0^{\circ}$ refers to the position on the $X Z$ plane whose velocity components along the $X$ and $Z$ axes are zero. Figure 5 presents the stable manifold passing perigee for the first and second times, while the lowest heights of these two families are highlighted with the green and blue thick dashed lines. That means there are several nearly zero-energy transfer opportunities with the help of the stable manifold. The window will expand if a small injection velocity is applied to adjust the perigee altitude. However, some transfers correspond to large speed increments considering the ephemeris, in which case, for more stable manifolds passing through the Earth's perigee, more times will be considered.

In order to enable the four detectors and the collector to form the configuration and implement the observation as soon as possible after the launch, the three launches are designed to be completed in one orbital period, which can maximize the observation mission time during the lifetime of the satellites. Based on the analysis of CRTBP, we record all the perigees of the manifold under the ephemeris model whose transition time is within two halo orbital periods and select the solution with less cost to adjust the height of the perigee. At the same time, the time interval between each launch is restricted to more than three months considering ground constraints.

The parameters of the three launches are presented in Table 3. The interval of each launch is larger than three months, and the whole three velocity increments for insertions are less than $30 \mathrm{~m} / \mathrm{s}$. The initial velocity increment from the parking orbit to the stable manifolds is about $3.19 \mathrm{~km} / \mathrm{s}$. As per the statement above, we choose the stable manifold of the $1 \mathrm{st}, 4$ th, and 7 th perigee pass-through to minimize the insertion cost. The three transfers in the Earth J2000 and the rotating frame are shown in Figure 6. The total transfer time from the first launch to the last insertion is about 543 days, and the total velocity increment can meet the mission requirement.

We further change the first insertion time to see the influence of time on the launch state. It is concluded that the time of the mission has little influence on the velocity increment of the transfers, for the initial velocity increments at different times are almost the same, and the insertion velocity increments for the adjustment of the perigee height are very small with the order of $\mathrm{m} / \mathrm{s}$.

\section{Impulsive-Based Control for Formation Maintenance}

Due to the strong nonlinearity of the dynamics near libration points, the formation nearby needs to be maintained to resist divergence. The required accuracy of relative distances between each detector and the collector is given in Table 1 during the mission execution. Therefore, control is required to maintain the normal execution of the observation mission in the natural evolution of configuration when the relative position exceeds the constraint level. Several kinds of control methods are investigated in recent years, such as the LQR method; the improved polynomial eigenstructure assignment (PEA) method [15]; a multiagent, nonlinear, and constrained optimal control method [16]; and the geometric control method [17].

The abovementioned continuous control method is applicable near the equilibrium point sensitive to small disturbances. However, in some scenarios, it is necessary to use an impulse control strategy while excluding continuous control to meet specific mission constraints. The aiming method based on the differential correction scheme called the equitime targeting method (ETM) proposed by Barden and Howell [7] is a widely adopted strategy. The nominal path is divided into segments of a given time, and impulse maneuvers are performed with the nominal state at the end of each segment as the target. The fixed time interval leads to waste in some trajectory sections with small errors, and the unnecessary switch on and off will also cause unnecessary engine loss. In 2012, Qi et al. [18] proposed the tangent targeting method (TTM) based on a two-level differential correction, which could fully and efficiently satisfy the predetermined error bound. The comparison between the TTM and ETM shows that the number of maneuvers can be significantly reduced and the length of time between successive maneuvers can be greatly increased. 
TABle 3: Parameters of three transfers.

\begin{tabular}{lccccc}
\hline Group & Launch time & $\begin{array}{c}\text { Initial } \Delta V \\
(\mathrm{~km} / \mathrm{s})\end{array}$ & Insertion time & $\begin{array}{c}\text { Insertion } \Delta V \\
(\mathrm{~m} / \mathrm{s})\end{array}$ & $\begin{array}{c}\text { Insertion phase } \\
(\mathrm{deg})\end{array}$ \\
\hline 1st & $2026-12-26$ & 3.1900 & $2028-05-16$ & 7.60 & 281 \\
2nd & $2027-06-03$ & 3.1903 & $2028-06-21$ & 0.023 & 353 \\
3rd & $2027-12-11$ & 3.1905 & $2028-06-10$ & 2.20 & 331 \\
\hline
\end{tabular}

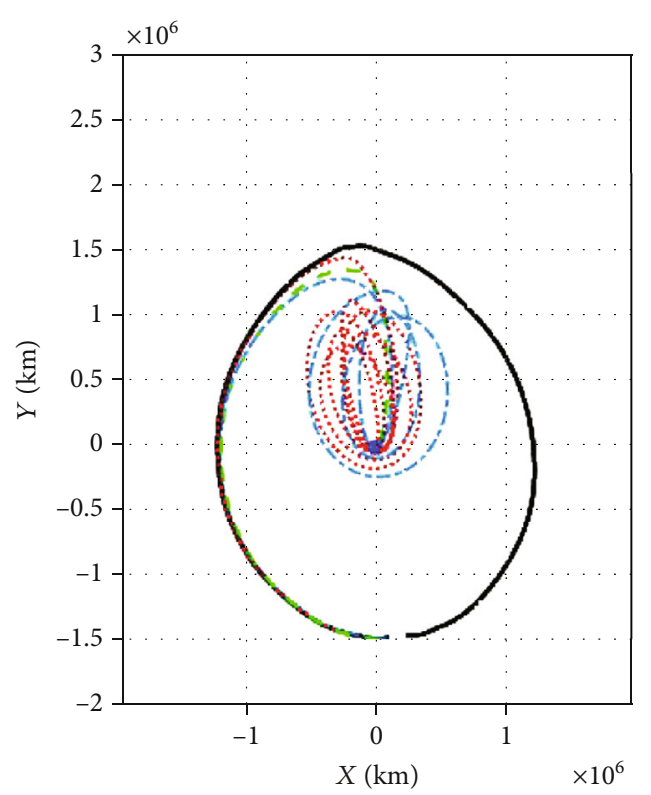

(a)

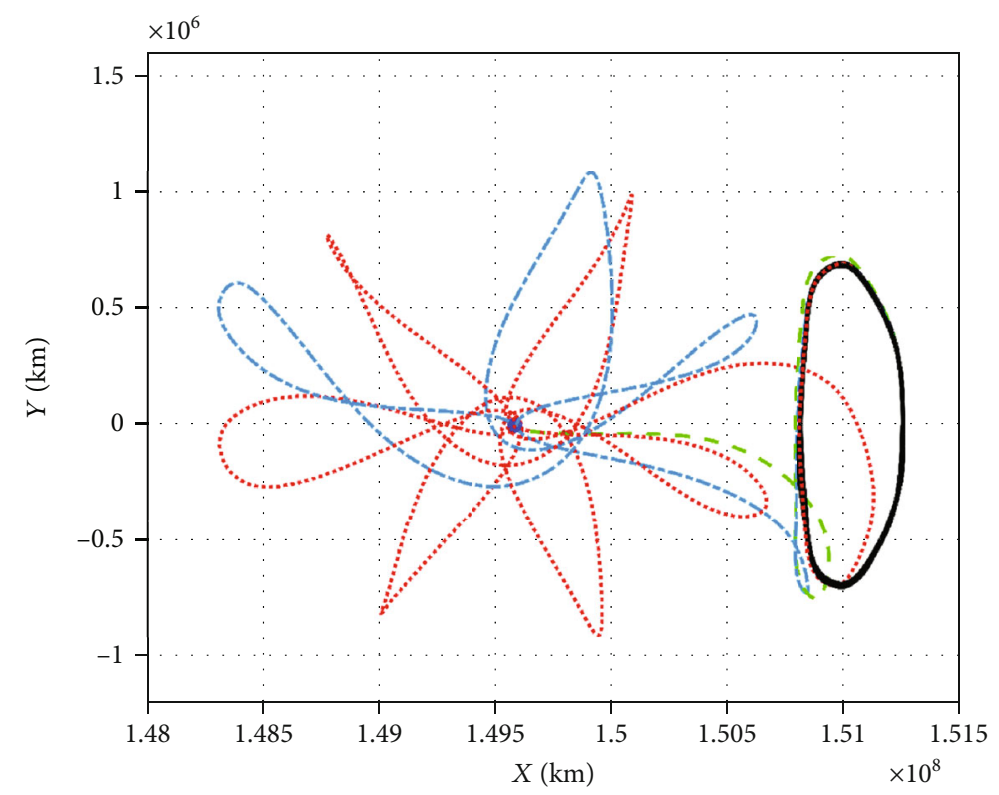

(b)

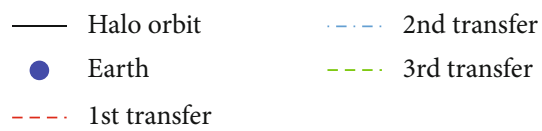

FIgURE 6: Transfer trajectories and mission orbit for exoplanet exploration: (a) Earth J2000; (b) Sun-Earth rotating frame.

For the maintenance of the formation configuration of the libration point, the constraint of maximum drift error bound exists in this project, so the control law of TTM [18] is more applicable, which can maximize the time spent within the error bound between maneuvers. Figure 7 shows the definition of the square configuration direction in the rotating system and the configuration parameters of the formation. Let $\mathbf{n}$ be the normal of the formation, and its direction is defined by two angles $\alpha, \beta$ in the spherical coordinates. $\beta$ is the angle between $\mathbf{n}$ and axis $O z, \alpha$ is the angle between the projection of $\mathbf{n}$ on plane $O x y$ and axis $O x$, and $C$ is the collector located at the centroid. The globe with the rotating system is used to facilitate the representation direction of the configuration, and the little tiny blue body in the globe represents the Earth. In Figure 7, the positions of the four detectors are noted as $\mathbf{r}_{1}, \mathbf{r}_{2}, \mathbf{r}_{3}, \mathbf{r}_{4}$, and the size of the configuration is defined by the lengths of the four arms

$$
L_{1}=\left|\mathbf{r}_{1}\right|, L_{2}=\left|\mathbf{r}_{2}\right|, L_{3}=\left|\mathbf{r}_{3}\right|, L_{4}=\left|\mathbf{r}_{4}\right|
$$

At the beginning of the mission, the size of the configuration is given by

$$
L_{1}=L_{2}=L_{3}=L_{4}
$$

When the formation propagates on the mission orbit, due to the perturbation, four detectors gradually deviate from their nominal positions relative to the collector, causing the deformation of the configuration. Through previous research [19], it is found that when the initial configuration plane near the libration point points to the $X$-axis of the rotating system, the stability of the formation is best. Therefore, in this mission, the positions of four detectors relative to the collector in the rotating system are selected to make the configuration plane point to the $X$-axis of the rotating system, which indicates that

$$
\alpha=0^{\circ}, \beta=90^{\circ} \text {. }
$$

For a space-based exoplanet exploration mission, it is required that each detector is located at a fixed position 


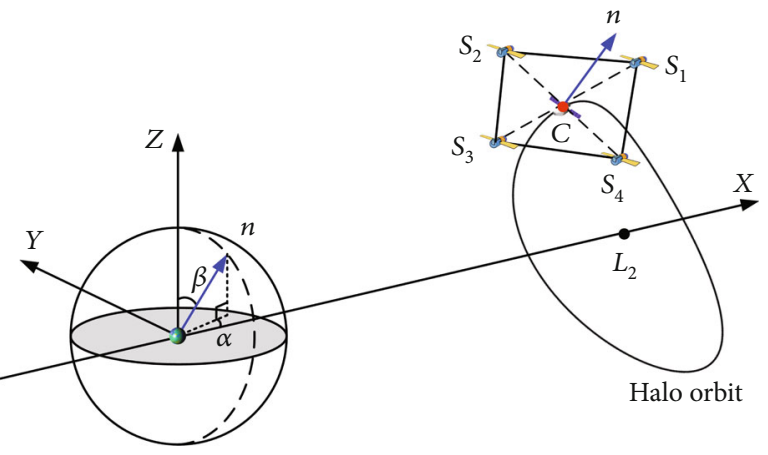

(a)

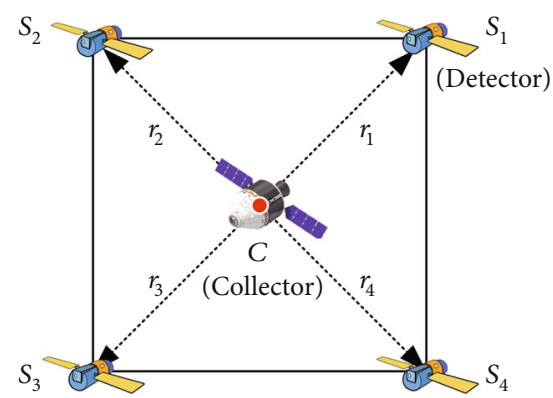

(b)

Figure 7: Square configuration of the formation: (a) direction definition of the formation; (b) arms of the formation.

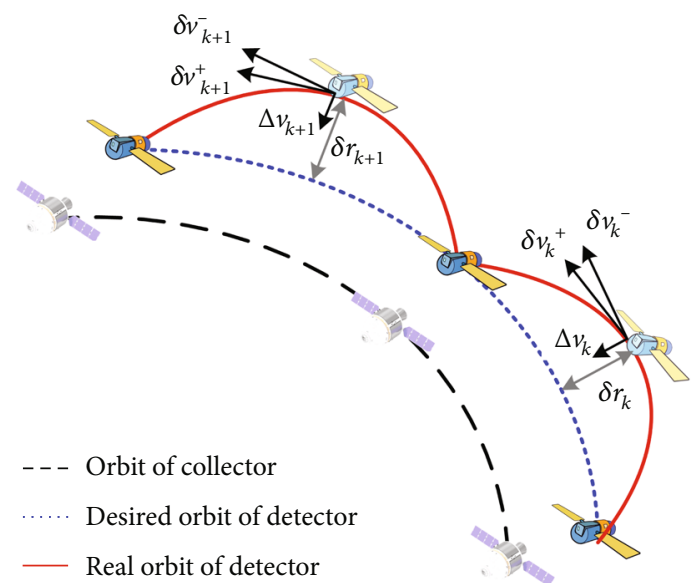

Figure 8: Principle of tangent targeting method.

relative to the detector. That is to say, the detectors' dynamics are modeled as a perturbation relative to the motion of the collector:

$$
\delta \mathbf{r}(t)=\delta \mathbf{r}_{0},
$$

where $\delta \mathbf{r}_{0}$ is the desired relative position.

As is shown in Figure 8, the desired path of the detector is divided into segments of a given error bound. The start and the end of a segment are noted as

$$
T_{k}=\left[t_{k}, t_{k}+\Delta t\right]
$$

where $\Delta t$ is the time spent of the considered segment. Assume that the relative state of the collector is

$$
\delta \tilde{\mathbf{X}}_{k}=\left[\delta \tilde{\mathbf{r}}_{k} ; \delta \tilde{\mathbf{v}}_{k}^{-}\right] .
$$

The desired orbit is satisfied at the start $t_{k}$ of the segment, $\delta \tilde{\mathbf{r}}_{k}=\delta \mathbf{r}_{0}$.

According to the Taylor expansion, in segment Equation (5), we have

$$
\delta \tilde{\mathbf{r}}\left(t_{k}+t_{f}\right)=\delta \tilde{\mathbf{r}}_{k}+\delta \tilde{\mathbf{v}}_{k} t_{f}+\frac{1}{2}\left(\mathbf{A} \delta \tilde{\mathbf{r}}_{k}+\mathbf{C} \delta \tilde{\mathbf{v}}_{k}\right) t_{f}^{2}
$$

We substitute $t_{f}=\Delta t$ into Equation (7) and set $\delta \tilde{\mathbf{r}}\left(t_{k}+\right.$ $\Delta t)=\delta \mathbf{r}_{0}$, then

$$
\delta \mathbf{v}_{k}^{+}=-\frac{1}{2}\left[\mathbf{I}_{3} \Delta t_{k}+\frac{1}{2} \mathbf{C} \Delta t^{2}\right]^{-1} \mathbf{A} \delta \mathbf{r}_{k} \Delta t^{2} \approx-\frac{1}{2} \mathbf{A} \delta \mathbf{r}_{0} \Delta t,
$$

where $\delta \mathbf{v}_{k}^{+}$is the velocity at the start of the segment after impulsive control, $\delta \mathbf{v}_{k}^{+}=\delta \mathbf{v}_{k}^{-}+\Delta v_{k}$.

After preliminary analysis, the $k$-th time span $\Delta t_{k}$ for orbit maintenance can be approximately given by

$$
\Delta t_{k}=\sqrt{\frac{8 \varepsilon_{\max }}{\left\|\mathbf{A} \delta r_{k}\right\|}},
$$

where $\varepsilon_{\max }$ is the allowed error bound, and then, the correction equation after derivation can be formulated as

$$
\Delta t^{s+1}=\Delta t^{s}+\frac{4}{\Delta t^{s}\left\|\mathbf{A} \delta r_{k}\right\|}\left(\varepsilon_{\max }-\varepsilon^{s}\right),
$$

where $\varepsilon^{s}$ is the maximum position error in the natural propagating time corresponding to the $s$-th iteration for $k$-th orbit maintenance.

Here, we assume four detectors of $500 \mathrm{~kg}$ form a square formation with the size of $200 \mathrm{~m}$, and the halo orbit with an amplitude of $150,000 \mathrm{~km}$ is chosen as the mission orbit. The relative positions of the four detectors are

$\mathbf{r}_{1}=[0,0,200] \mathrm{m}, \mathbf{r}_{2}=[0,200,00] \mathrm{m}, \mathbf{r}_{3}=[0,0,-200] \mathrm{m}, \mathbf{r}_{4}=[0,-200,0] \mathrm{m}$.

The tolerance of position error is set as $\delta \mathbf{r}_{k}=0.1 \mathrm{~m}$ throughout the whole mission. The control quantity and control deviation of the four detectors are shown in Figure 9.

Due to the symmetry of the natural drift of the four detectors, the control forces of $S_{1}$ and $S_{3}$ located in the positive and negative directions of the $Z$-axis have opposite directions, and the magnitude and application time are the same, while $S_{2}$ and $S_{4}$ present the same features. In a mission orbit period of about 180 days, 92 impulses need to be applied to $S_{2}$ and $S_{4}$, and the whole velocity increments are both $4.30 \times 10^{-4} \mathrm{~m} / \mathrm{s}$, while 99 impulses apply to $S_{1}$ and $S_{3}$ with $4.99 \times 10^{-4} \mathrm{~m} / \mathrm{s}$ velocity increment. It is shown that 

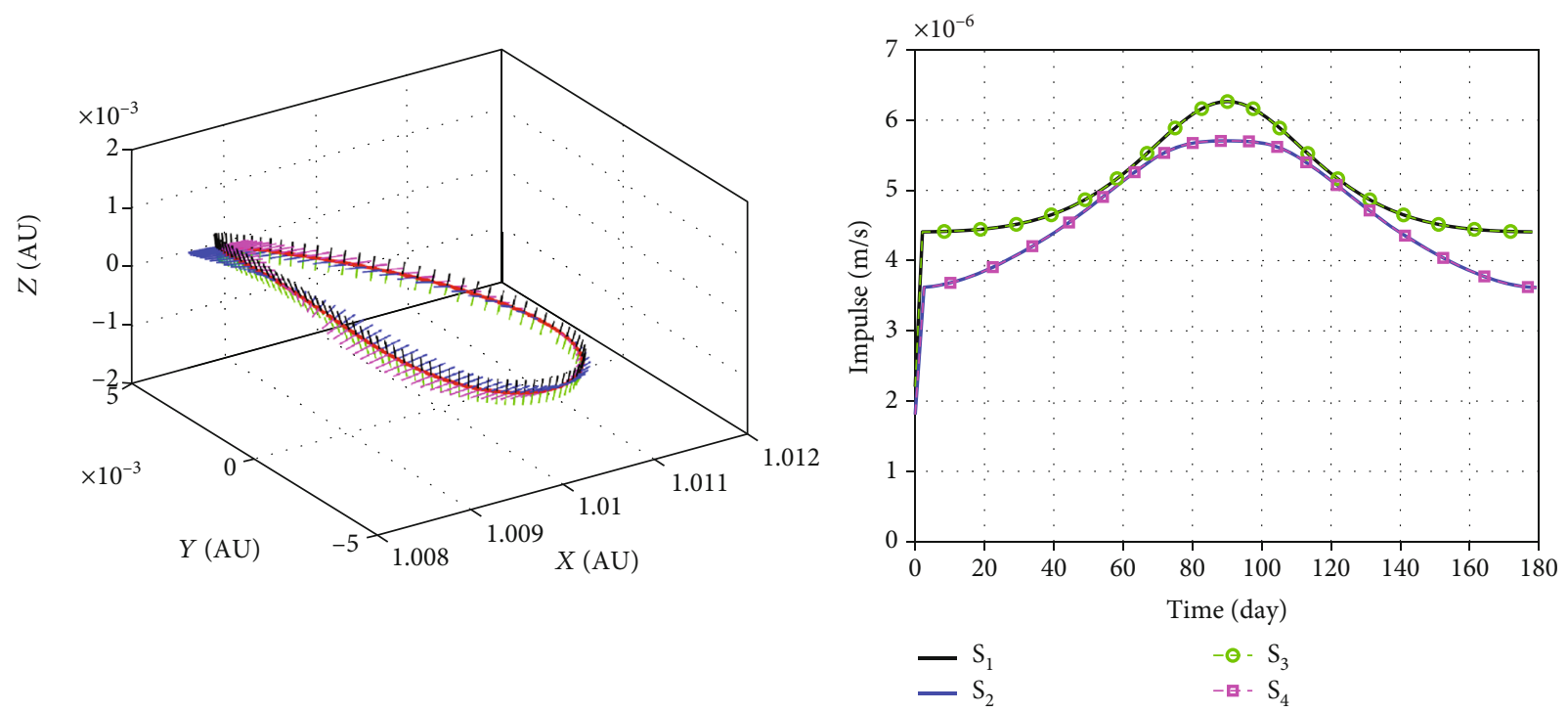

(a)

(b)

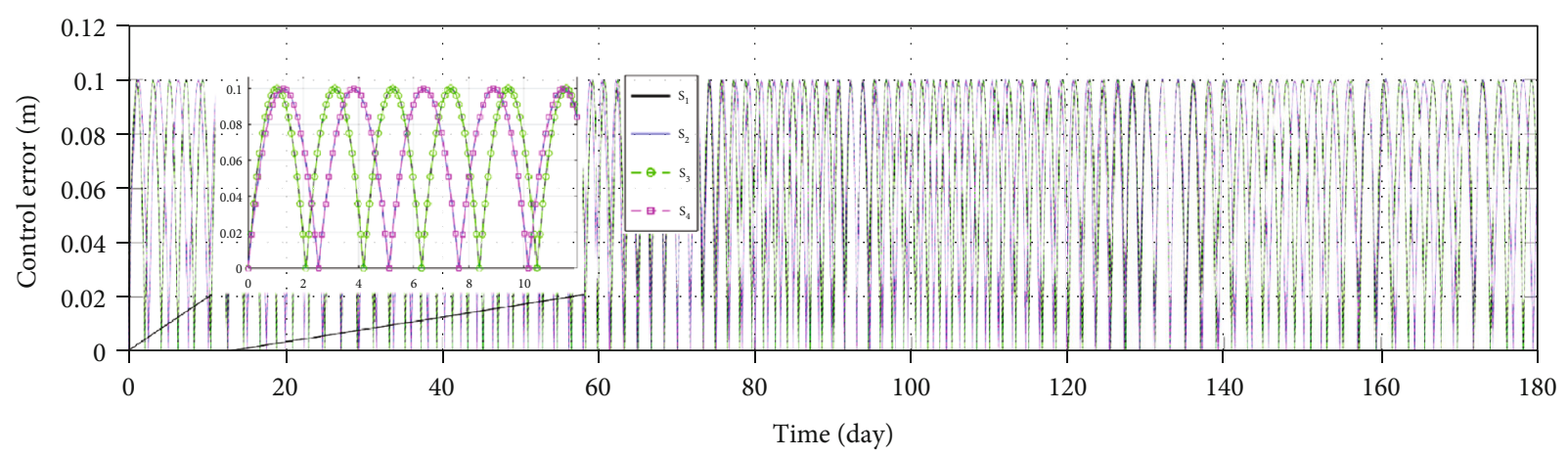

(c)

FIgURE 9: Parameters of formation control using TTM: (a) control vectors; (b) control amplitude; (c) control error.

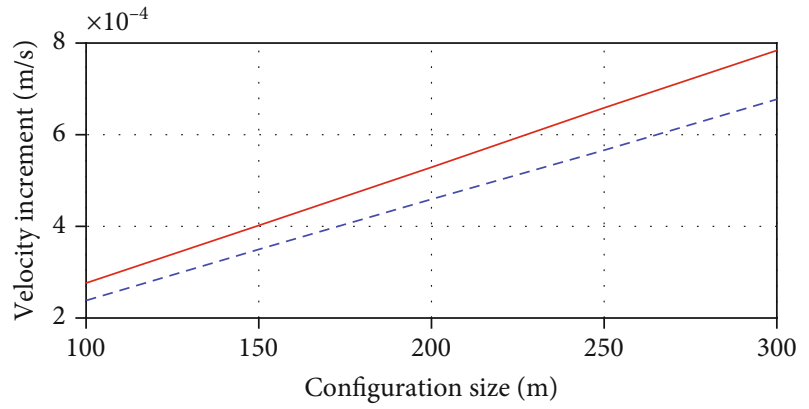

(a)

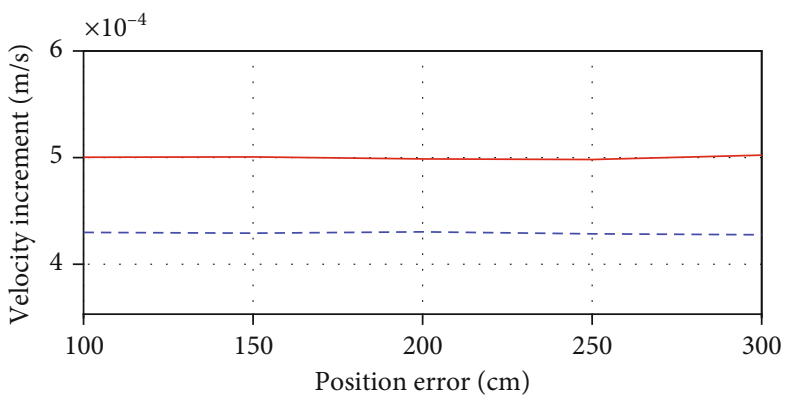

(b)

- Detectors $1 \& 3$

- - - Detectors $2 \& 4$

FIGURE 10: Velocity increments in two conditions of TTM: (a) different relative position errors; (b) different formation sizes.

the control strategy can well meet the relative position stability constraints of the configuration.

The effects of formation sizes and relative position accuracy on the fuel consumption are further discussed. Figure 10 shows the velocity increments required for TTM control in two cases: (a) The relative position error bound is fixed at $10 \mathrm{~cm}$, and the formation configuration size varies between $100 \mathrm{~m}$ and $300 \mathrm{~m}$. (b) The formation configuration size is $200 \mathrm{~m}$, and the relative position error bound varies between $1 \mathrm{~cm}$ and $20 \mathrm{~cm}$.

It can be seen that the velocity increment required for formation position control is not affected by formation size 
but has a linear relationship with the relative position error bound between the detectors and the collector. Therefore, the formation size is a design index that needs to be considered when considering fuel consumption constraints, and the position error tolerance can be selected according to the onboard hardware constraints and the desired control frequency.

\section{Conclusion}

This paper carried out the mission design of the space-based exoplanet exploration by an aperture-synthetic interferometer system. First, the system architecture and mission profile are discussed. Then, considering the lighting and thermal conditions and the cost of orbital maintenance, the SunEarth L2 halo orbit is selected as the mission orbit. The low-energy transfer by the stable manifold is designed under the ephemeris model. The multiple perigees of the manifold are found to reduce the total transfer time to one and a half years. Each transfer only needs a velocity increment less than $10 \mathrm{~m} / \mathrm{s}$ to achieve the insertion of the halo orbit. Finally, TTM is applied to maintain the configuration of the formation drifting in the natural state, which can efficiently complete the high-precision maintenance of the formation under the constraint of position accuracy. The spacecraft can satisfy the relative position stability constraints at a maintenance frequency of once every two days approximately, with the whole velocity increments of each spacecraft are less than $5 \times 10^{-4} \mathrm{~m} / \mathrm{s}$ when the error bound is $0.1 \mathrm{~m}$. Both the trajectory design and orbital maintenance can meet the mission requirement. The study will provide a design reference for future space-based exoplanet exploration missions.

\section{Data Availability}

The data used to support the findings of this study are available from the author upon reasonable request.

\section{Conflicts of Interest}

The authors declare that there is no conflict of interest regarding the publication of this paper.

\section{Authors' Contributions}

Feida Jia and Xiangyu Li contributed to the literature review, the simulation, and the writing and revision of the paper. Zhuoxi Huo performed the literature review, the mission architecture, and the principle of interferometry. Dong Qiao contributed to the validation of the method and revised this paper.

\section{Acknowledgments}

This work was supported by the National Natural Science Foundation of China (Grant Nos. 12002028 and 51827806) and Beijing Institute of Technology Research Fund Program for Young Scholars.

\section{References}

[1] N. A. Bahcall and A. Burrows, "Profile of James Peebles, Michel Mayor, and Didier Queloz: 2019 Nobel Laureates in Physics," Proceedings of the National Academy of Sciences of the United States of America, vol. 117, no. 2, pp. 799-801, 2020.

[2] R. J. Malcuit, "Discussion of the probability of finding habitable planets for humans orbiting sun-like stars," in The Twin Sister Planets Venus and Earth, Springer, Cham, 2015.

[3] D. R. Coulter, NASA's terrestrial planet finder mission: the search for habitable planets, European Space Agency, (Special Publication) ESA SP, 2003.

[4] M. Fridlund and L. Kaltenegger, ESA's search for ectra-solar terrestrial planets: mission update of the Darwin Project, European Space Agency, (Special Publication) ESA SP, 2002.

[5] K. G. Carpenter, C. J. Schrijver, M. Karovska, and SI Mission Concept Development Team, "The stellar imager (SI) project: a deep space UV/optical interferometer (UVOI) to observe the universe at 0.1 milli-arcsec angular resolution," Astrophysics and Space Science, vol. 320, no. 1-3, pp. 217-223, 2009.

[6] Z. Tong, M. Li, C. Cui, Z. Huo, and B. Luo, "Design and analysis of the configuration of deployable membrane sunshield," Zhongguo Kongjian Kexue Jishu/Chinese space Science and Technology, vol. 41, no. 3, p. 82, 2021.

[7] B. T. Barden and K. C. Howell, "Formation flying in the vicinity of libration point orbits," Advances in the Astronautical Sciences, vol. 99, no. 2, pp. 969-988, 1998.

[8] A. Héritier and K. C. Howell, "Dynamical evolution of natural formations in libration point orbits in a multi-body regime," Acta Astronautica, vol. 102, pp. 332-340, 2014.

[9] A. Héritier and K. C. Howell, "Natural regions near the collinear libration points ideal for space observations with large formations," The Journal of the Astronautical Sciences, vol. 60, no. 1, pp. 87-108, 2013.

[10] M. Xu, Y. Liang, and X. Fu, "Formation flying on quasi-halo orbits in restricted Sun-Earth/Moon system," Aerospace Science and Technology, vol. 67, pp. 118-125, 2017.

[11] A. Kutlu and O. Tekinalp, "Formation flight design near sunearth collinear libration points under the effect of disturbances," in 2019 9th International Conference on Recent Advances in Space Technologies (RAST), pp. 137-144, Istanbul, Turkey, 2019.

[12] W. Wang, H. Baoyin, G. Mengali, and A. A. Quarta, "Solar sail cooperative formation flying around L2-type artificial equilibrium points," Acta Astronautica, vol. 169, pp. 224239, 2020.

[13] T. Velusamy, R. P. Angel, A. Eatchel, D. Tenerelli, and N. J. Woolf, "Single and double Bracewell nulling interferometer in space," European Space Agency, (Special Publication) ESA SP, vol. 2003, no. 539, pp. 631-636, 2003.

[14] E. Di Sotto, L. Tarabini, and M. Graziano, "Heliocentric earth trailing orbit design for a small probe concept," Advances in the Astronautical Sciences, vol. 114, pp. 2063-2073, 2003.

[15] F. Wang, X. Chen, A. Tsourdos, B. A. White, and Y. Wu, "Nonlinear relative position control of precise formation flying using polynomial eigenstructure assignment," Acta Astronautica, vol. 68, no. 11-12, pp. 1830-1838, 2011.

[16] S. I. Infeld, S. B. Josselyn, W. Murray, and I. M. Ross, "Design and control of libration point spacecraft formations," Journal of Guidance, Control, and Dynamics, vol. 30, no. 4, pp. 899909, 2007. 
[17] H. Peng, J. Zhao, Z. Wu, and W. Zhong, "Optimal periodic controller for formation flying on libration point orbits," Acta Astronautica, vol. 69, no. 7-8, pp. 537-550, 2011.

[18] R. Qi, S. Xu, and M. Xu, "Impulsive control for formation flight about libration points," Journal of Guidance, Control, and Dynamics, vol. 35, no. 2, pp. 484-496, 2012.

[19] F. Ferrari and M. Lavagna, "Suitable configurations for triangular formation flying about collinear libration points under the circular and elliptic restricted three-body problems," Acta Astronautica, vol. 147, pp. 374-382, 2018. 